\title{
High-quality continuous random networks
}

\author{
G. T. Barkema* \\ Theoretical Physics, Utrecht University, Princetonplein 5, 3584 CC Utrecht, the Netherlands \\ Normand Mousseau ${ }^{\dagger}$ \\ Department of Physics and Astronomy and CMSS, Ohio University, Athens, Ohio 45701
}

(Received 4 November 1999; revised manuscript received 25 April 2000)

\begin{abstract}
The continuous random network (CRN) model is an idealized model for perfectly coordinated amorphous semiconductors. The quality of a CRN can be assessed in terms of topological and configurational properties, including coordination, bond-angle distributions, and deformation energy. Using a variation on the sillium approach proposed 14 years ago by Wooten, Winer, and Weaire, we present 1000-atom and 4096-atom configurations with a degree of strain significantly less than the best CRN available at the moment and comparable to experimental results. The low strain is also reflected in the electronic properties. The electronic density of state obtained from $a b$ initio calculation shows a perfect band gap, without any defect, in agreement with experimental data.
\end{abstract}

\section{INTRODUCTION}

The structure of amorphous semiconductors, as seen by theorists, is well represented by the continuous random network (CRN) model introduced more than 60 years ago by Zachariasen. ${ }^{1}$ The interest of this model lies in its simplicity: the only requirement of this model is that each atom should satisfy fully its bonding needs. In addition, the quality of a CRN is generally determined by the amount of strain, as measured by the local deviations from crystalline environment, induced by this constraint; the "ideal" CRN being typically defined as that with the lowest spread in the bondlength and bond-angle distributions.

In spite of the simplicity of the model, it has turned out to be difficult to actually prepare CRN realizations of a quality comparable to that of experiment, making it difficult to fully assess the real structure of amorphous semiconductors. The origin of this problem has generally been attributed to weaknesses in the model-building community: standard approaches such as molecular dynamics cannot reach time scales appropriate for full relaxation. Moreover, other techniques suggest that empirical and semiempirical potentials able to reproduce all properties of amorphous semiconductors are still missing, ${ }^{2-4}$ although the introduction of environment-dependent potentials has improved the situation. ${ }^{5}$ An alternative explanation for the fact that experimentally a lower spread in bond-length and -angular distribution is observed might be that the coordination in highquality $a$-Si samples is significantly lower than four. Laaziri et al. ${ }^{6}$ report a coordination as low as 3.88, a density of defects at least an order of magnitude higher than what is measured using differential scanning calorimetry and electron-spin resonance techniques (which give, respectively, a $1 \%$ and $0.04 \%$ density of defects). ${ }^{7}$ If true, this higher density of defects might easily facilitate a lower spread in the bond lengths and angles, explaining in part the discrepancy between experiment and theoretical models.

Following a long tradition, one approach to shed some light on this discrepancy is to try to see how far it is possible to push the continuous random model in order to reach structural properties in agreement with experiment. By creating idealized networks with the same angular deviation as the experimental ones and a good overall fit to the radial distribution function, it is possible to show that perfect coordination in amorphous silicon is not ruled out by the low angular deviation. This is the purpose of this paper, which follows a long series of works with the same motivation. ${ }^{8-11}$

Using a modified version of the Wooten-Winer-Weaire (WWW) algorithm, we have succeeded in creating a number of totally independent 1000-atom configurations with a bond-angle distribution as low as $9.19^{\circ}$, almost $2^{\circ}$ below the best available numerical models without four-membered rings and on a par with experimental values. ${ }^{12}$ The algorithm we use avoids completely the crystalline state, contrary to previous WWW-type approaches. Overall, the excellent structural and electronic properties of the networks, including a totally clean electronic band gap, make them ideal starting point for empirical as well as tight-binding or $a b$

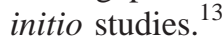

This paper is constructed as follows. First, we review briefly the Wooten-Winer-Weaire algorithm and detail our simulation procedure. Next, we present structural and electronic properties of the configurations generated, and compare them with previous simulations and experimental results.

\section{METHODOLOGY AND DETAILS OF SIMULATIONS}

In the sillium approach, ${ }^{9}$ proposed by Wooten, Winer, and Weaire to generate CRN structures, a configuration consists of the coordinates of all $N$ atoms, together with a list of the $2 N$ bonds between them. The structural evolution consists of a sequence of bond transpositions involving four atoms. Four atoms $A, B, C$, and $D$ are selected following the geometry shown in Fig. 1; two bonds, $A B$ and $C D$, are then broken, and atoms $A$ and $D$ reassigned, respectively, to $C$ and $B$, creating two new bonds, $A C$ and $B D$. After the transposi- 


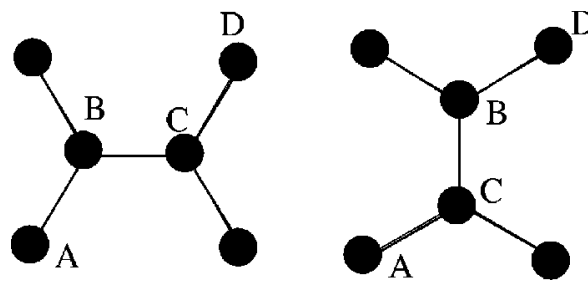

FIG. 1. A basic WWW event. Left: before; right: after the bond exchange.

tion, all atoms are allowed to relax within the constraints of the neighbor list.

Within this approach, the generation of a CRN starts with a cubic periodic diamond structure, which is then randomized by a large number of bond transpositions. After thermalization, the network is relaxed through a sequence of many more proposed bond transpositions, accepted with the Metropolis acceptance probability

$$
P=\operatorname{Min}\left[1, \exp \left[\left(E_{b}-E_{f}\right) / k_{B} T\right]\right],
$$

where $k_{B}$ is the Boltzmann constant, $T$ is the temperature, and $E_{b}$ and $E_{f}$ are the total energies of the system before and after the proposed bond transposition.

The list of neighbors determines the topology, but also the energy of the network: independently of the distance between two atoms, they interact only if they are connected in the list of neighbors. With an explicit list of neighbors, it is possible to use a simple interaction such as the Keating potential: ${ }^{14}$

$$
E=\frac{3}{16} \frac{\alpha}{d^{2}} \sum_{\langle i j\rangle}\left(\mathbf{r}_{i j} \cdot \mathbf{r}_{i j}-d^{2}\right)^{2}+\frac{3}{8} \frac{\beta}{d^{2}} \sum_{\langle j i k\rangle}\left(\mathbf{r}_{i j} \cdot \mathbf{r}_{i k}+\frac{1}{3} d^{2}\right)^{2},
$$

where $\alpha$ and $\beta$ are the bond-stretching and bond-bending force constants and $d=2.35 \AA$ is the $\mathrm{Si}$-Si strain-free equilibrium bond length in the diamond structure. Usual values are $\alpha=2.965 \mathrm{eV} / \AA^{2}$ and $\beta=0.285 \alpha$.

With the approach described above, along with a few more details that can be found in Ref. 9, Wooten and Weaire obtained 216-atom structures with an angular distribution as low as $10.9^{\circ}$. A decade later, using the same approach but more computing power, Djordjevic, Thorpe, and Wooten (DTW) produced some large (4096-atom) networks of even better quality, with a bond-angle distribution of 11.02 degrees for configurations without four-membered rings and $10.51^{\circ}$ when these rings where allowed. ${ }^{11}$

In the present work, using a series of algorithmic improvements and faster computers, we are able to generate structurally and electronically better networks: the 1000atom configurations, for example, show a bond-angle distribution of almost $2^{\circ}$ lower than DTW's model while our 4096-atom cell is more than $1^{\circ}$ better.

The improvements introduced to the sillium approach are the following:

(1) We start from a truly random configuration rather than from a molten crystalline state, thus guaranteeing that the structure is not contaminated by some memory of the crystalline state.

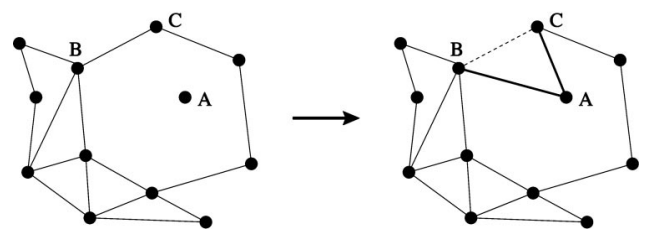

FIG. 2. One step in the expansion of the loop, that eventually visits all atoms four times. Three atoms $A, B$, and $C$ are involved, of which $B$ and $C$ are bonded, while $A$ is bonded to neither $B$ nor $C$, and of which $A$ is not fourfold coordinated. The bond $B C$ is then replaced by bonds $A B$ and $A C$.

(2) We evaluate the acceptance of a trial move using a Metropolis accept/reject procedure without doing full relaxation.

(3) We use a local/nonlocal relaxation procedure to limit the number of force evaluations, i.e., we relax only locally in the first ten relaxation steps after a bond transposition (up to the third neighbor shell); in combination with improvement (2), this makes the time per bond transposition almost independent of the configuration size.

(4) At regular times, we quench the structure at zero temperature, with advantages outlined in Sec. II C.

With these improvements, the generation of the networks proceeds as follows. We first generate starting configurations as described in Sec. II A, and quench these structures as described in Sec. II C. Next, we alternate running at a temperature of $0.25 \mathrm{eV}$ for about 100 trial bond transpositions per atom and quenching. The decrease in energy is almost exclusively obtained during the quenching, the role of the annealing at finite temperature is mostly to provide for a fresh starting point for the next quench. Once the energy is brought down to about $0.3 \mathrm{eV}$ per atom, and the angular spread around $10^{\circ}$, this procedure yields diminishing returns: the annealing is no longer able to bring the configuration to a starting point where the quenching leads to a lower minimum. To lower the energy further, we therefore also anneal the configurations in different conditions for a few hundred trial bond transpositions per atom, like a stronger three-body force or a larger volume.

\section{A. Generating random initial CRN's}

To generate a random initial configuration, we randomly place the atoms in a cubic box with periodic boundary conditions at crystalline density, under the constraint that no two atoms be closer than $2.3 \AA$. The difficult part is to connect these atoms in order to obtain a tetravalent network. We achieve this by starting with a loop visiting four atoms somewhere in the configuration, in such a way that each pair of atoms that are neighbors along the loop be not separated by more than a cutoff distance $r_{c}$. This loop is gradually expanded until it visits each atom exactly twice; the steps of the loop are then the bonds in our tetravalent network. The expansion of the loop is achieved by randomly selecting a group of three atoms $A, B$, and $C$, such that $A$ is not fourfold coordinated and is within a distance of $r_{c}$ from $B$ and $C$ but not bonded to either, while $B$ and $C$ are bonded. Next, the bond $B C$ is replaced by bonds $A B$ and $A C$, expanding the loop by one step. This procedure is illustrated in Fig. 2. Initially, $r_{c}$ is set to some small value like $3 \AA$, but then it is 
gradually increased until all atoms are fourfold coordinated. Although this method leads to highly strained initial configurations, it has the advantage that it contains absolutely no trace of crystallinity.

This process leads typically to CRN's whose angular distribution initially has a width of around $30^{\circ}$, but which reduces rapidly to around $13^{\circ}$ in a single quench (see Sec. II C). In the beginning of this first quench, when the angular deviation is quite large, sometimes a pair of atoms is close by without being bonded; to eliminate such artifacts that result from the fact that within the Keating potential atoms only interact if they are explicitly bonded, we replace a bond of each of these atoms by a bond between these atoms and another bond between their neighbors (conserving four-fold coordination).

\section{B. Avoiding complete relaxation of trial configurations}

In the standard sillium approach, a move consists of a bond transposition followed by full structural relaxation and an accept/reject step according to the Metropolis criterion (1). An alternative implementation is to first decide a threshold energy given by

$$
E_{t}=E_{b}-k_{B} T \ln (s),
$$

where $s$ is a random number between 0 and 1 . The proposed move is then accepted only if $E_{f} \leqslant E_{t}$. This procedure is exactly equivalent to the usual Metropolis procedure. By fixing the threshold for $E_{f}$ before a transposition is attempted, it is however possible to reject the move as soon as it becomes clear that this threshold cannot be reached, i.e., before the configuration is fully relaxed. Since the energy is harmonic around the minimum, the decrease in energy obtained during further relaxation is approximately equal to the square of the force times some proportionality constant $c_{f}$, so that during the relaxation the final energy can be estimated to be

$$
E_{f} \approx E-c_{f}|F|^{2} \text {. }
$$

If, at any moment during the relaxation, $E-c_{f}|F|^{2}>E_{t}$, the trial move is rejected and a new one is started. Such a method requires, of course, a conservative choice for $c_{f}$; in our units, the proportionality constant $c_{f}$ in well-relaxed configurations is always well below 1 . To account for anharmonicities, we do not reject any move during the first five steps of relaxation.

Since much less than $1 \%$ of the proposed moves are accepted in well-relaxed configurations, avoiding spending time on moves that are eventually rejected can produce a significant gain in efficiency; using this improvement, we observed an acceleration of close to an order of magnitude, so that all bond transpositions in a 1000-atom network can be attempted in less then $3 \mathrm{~min}$ on a $500 \mathrm{MHz}$ DEC-Alpha workstation.

\section{Efficient quenching}

Further optimizations are possible in the case of zero temperature. Since the threshold energy [Eq. (3)] is constant, a proposed bond transposition that is once rejected will keep on being rejected, as long as no other bond transpositions are accepted in the meantime. A combination of four atoms
TABLE I. Energetic and structural properties of models relaxed with the Keating potential. The first two models, DTW ${ }^{(1)}$ and $\mathrm{DTW}^{(2)}$ are the models prepared in Ref. 11 and refer, respectively, to a model with and without four-membered rings. Configurations $\mathrm{A}$ and $\mathrm{B}$ are 1000-atom models prepared according to the procedure described here and "4096" is a 4096-atom model prepared the same way. All three models are without four-membered rings. The ring statistics are for irreducible rings and $\rho_{0}$ is based on $r_{0}$ $=2.35 \AA$

\begin{tabular}{lccccc}
\hline \hline & DTW $^{(1)}$ & DTW $^{(2)}$ & Conf. A & Conf. B & 4096 \\
\hline $\mathrm{E}(\mathrm{eV}) /$ atom & 0.336 & 0.367 & 0.267 & 0.264 & 0.304 \\
$\rho / \rho_{0}$ & 1.000 & 1.000 & 1.043 & 1.040 & 1.051 \\
$\langle r\rangle / r_{0}$ & 0.996 & 0.997 & 0.982 & 0.982 & 0.980 \\
$\Delta r / r_{0}(\%)$ & 2.52 & 2.65 & 3.94 & 3.71 & 4.17 \\
$\langle\theta\rangle$ & 109.24 & 109.25 & 109.30 & 109.27 & 109.28 \\
$\Delta \theta$ & 10.51 & 11.02 & 9.21 & 9.20 & 9.89 \\
& & & & & \\
Rings/atom & & & & & \\
4 & 0.015 & 0.000 & 0.000 & 0.000 & 0.000 \\
5 & 0.491 & 0.523 & 0.472 & 0.480 & 0.490 \\
6 & 0.698 & 0.676 & 0.761 & 0.750 & 0.739 \\
7 & 0.484 & 0.462 & 0.507 & 0.515 & 0.467 \\
8 & 0.156 & 0.164 & 0.125 & 0.116 & 0.148 \\
9 & & & 0.034 & 0.033 & 0.035 \\
\hline \hline
\end{tabular}

$A B C D$ with bonds $A B, B C$, and $C D$ can be selected in $N$ $\times 4 \times 3 \times 3 / 2$ times, so there are $18 \mathrm{~N}$ possible bond transpositions. We mark all bond transpositions that were rejected since the last accepted bond transposition to avoid retrying these. Once all bond transpositions have been tried but rejected, the quenching is complete. At this stage, the system is not only at a local energy minimum (i.e., a point in phase space where the force is zero and all eigenvalues of the hessian are positive), but no single bond transposition can lower the energy. The configurations we discuss here have this property.

In the standard sillium approach, the creation of fourmembered rings is disallowed. Following DTW, ${ }^{11}$ we find that especially for quenching the relaxation is significantly helped by allowing for four-membered rings, because of the large extra number of pathways accessible to the system. At the end of the quenching, the few four-membered rings that are created can easily be removed one by one, by chosing the energetically most favorable bond transposition in which bond $A B$ is part of the four-membered ring (and where no new four-membered rings are introduced). Typically, the energy increases by less than $1 \mathrm{eV}$ per removed four-membered ring. The angular distribution narrows by a few hundredths of a degree by the removal of fourfold rings, but the radial distribution function (RDF) is not significantly changed.

\section{RESULTING CONFIGURATIONS}

We present here results for three different configurations: two 1000-atom cells and one with 4096 atoms. In Table I, we compare our configurations, relaxed with the Keating potential used in the modified WWW algorithm, with those of Djordjević, Thorpe, and Wooten. ${ }^{11}$ We also provide the irreducible ring statistics, i.e., those rings in which no two atoms 
TABLE II. Structural properties of our configurations after relaxation with the modified Stillinger-Weber (MSW) potential. Except for the 4096-atom configuration, the topology has remained unchanged (see text.) For comparison purposes, the total ring number per atom (including reducible ones) is also reported, as well as the energy after relaxation with the original Stillinger-Weber (SW) potential; molecular dynamics-prepared configurations give $-4.088 \mathrm{eV} /$ atom (Ref. 23)

\begin{tabular}{lccc}
\hline \hline & Conf. A & Conf. B & 4096 \\
\hline $\mathrm{E}(\mathrm{eV}) /$ atom (MSW) & -4.026 & -4.034 & -3.990 \\
$\mathrm{E}(\mathrm{eV}) /$ atom $(\mathrm{SW})$ & -4.126 & -4.133 & -4.106 \\
$\rho / \rho_{0}$ & 0.947 & 0.950 & 0.936 \\
$\langle r\rangle / r_{0}$ & 1.018 & 1.017 & 1.020 \\
$\Delta r / r_{0}(\%)$ & 2.9 & 2.7 & 3.2 \\
$\langle\theta\rangle$ & 109.25 & 109.24 & 109.20 \\
$\Delta \theta$ & 9.77 & 9.70 & 10.51 \\
& & & \\
Rings/atom & & & \\
4 & 0.000 & 0.000 & 0.001 \\
5 & 0.472 & 0.480 & 0.489 \\
6 & 0.840 & 0.847 & 0.830 \\
7 & 1.011 & 1.023 & 0.979 \\
8 & 2.025 & 2.002 & 2.064 \\
\hline \hline
\end{tabular}

are connected through a sequence of bonds that is shorter than the sequence along the ring. We also provide the ring statistics for all $n$-membered rings in order to compare with Ref. 9 and other papers in the literature (Table II). Table I shows that the strain per atom in our structures is significantly below that of DTW. One of the standard measurements to evaluate the quality of a model is the coordination number as computed based on the RDF. Using the minimum of the RDF between the first- and second-neighbor peak and after relaxation with the Keating model, the first two configurations are perfectly tetravalent. The 4096-atom configuration has $0.1 \%$ of five-fold defects. If our configurations are scaled to crystalline density, the energy increases by $6 \mathrm{meV}$ per atom.

Another important quantity that can be compared with experiment is the width of the bond-angle distribution. Experimentally this quantity can be extracted from the radial distribution function, or from the Raman spectrum-using a relation proposed by Beeman et al. ${ }^{15}$ The most recent measurement, taken on annealed samples prepared by ion bombardment and using the second-neighbor peak of the radial distribution function, gives $10.45^{\circ}$ and $9.63^{\circ}$, respectively, for as-implanted and annealed samples. ${ }^{12}$ Our configurations, relaxed with the Keating potential, present therefore a bondangle distribution slightly narrower than experimental values. (This is to be expected of the "right" structure since the theoretical models are taken at $0 \mathrm{~K}$, and since local relaxation with potentials more realistic than the Keating one results in wider angular distributions.)

While structural averages provide a good idea of the overall quality of a model, they do not say much regarding the local environments. It is therefore important to look also at the electronic properties of these models: even small densities of highly strained geometries or defect atoms will be picked up as states in the gap of the electronic density of
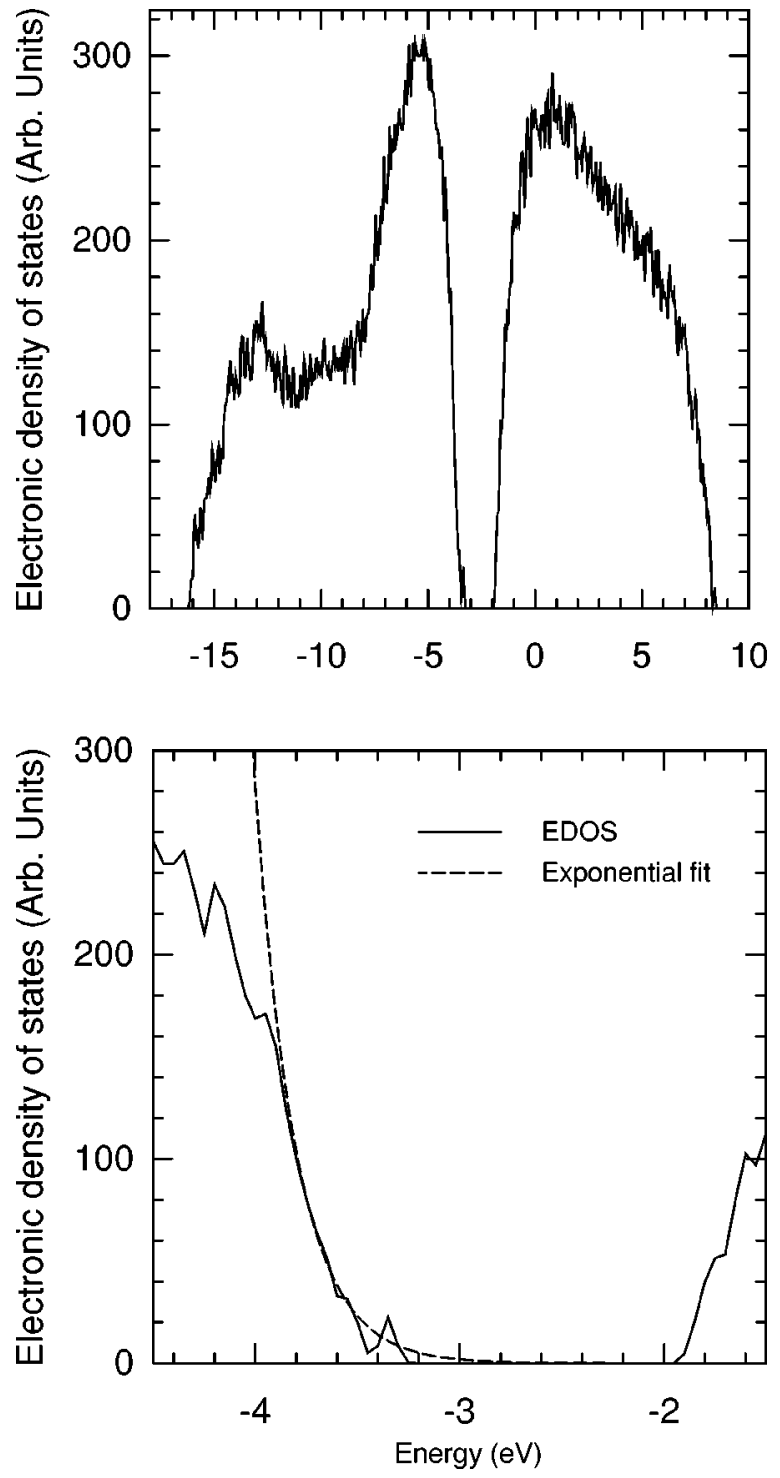

FIG. 3. Top: Electronic density of states for the 1000-atom configuration A as obtained using $a b$ initio tight binding. Bottom: Closeup of the gap region. The dashed curve is an exponential fit, $\rho(E) \propto \exp \left(-E / E_{0}\right)$, with $E_{0}=0.2 \mathrm{eV}$.

states (EDOS). In the last few years, it has become possible to compute the electronic structure of multi-thousand-atom configurations. Here, we show the electronic density of state for configuration A, a 1000-atom configuration. Because of the costs of doing a full $a b$ initio atomic relaxation, we have relaxed the cell with the Keating potential and used the FIREBALL local-basis ab initio code to obtain the electronic density of state. ${ }^{16}$ Previous work showed that configurations relaxed with a Keating potential demonstrated little further relaxation with the FIREBALL code ${ }^{17}$ so that the results presented here are unlikely to change very much during further relaxation. Figure 3 shows the EDOS smoothed with a Gaussian of width $0.01 \mathrm{eV}$. A remarkable feature of this EDOS is the absence of states in the gap, leading to a perfect gap of $1.3 \mathrm{eV}$. The generation of defectless models is very important for our understanding of the electronic dynamics and the role of defects in disordered semiconductors. The decay of the valence tail, the Urbach tails, can be reasonably well approximated by an exponential- 
$\rho(E) \propto \exp \left(-E / E_{0}\right)$-with $E_{0}=0.2 \mathrm{eV}$, in agreement with previous calculations. ${ }^{17}$

Although we get good structures using the Keating potential, it is important to verify the stability of these networks when relaxed with a more realistic interaction potential that does not require a preset list of neighbors. There exists no empirical potential at the moment that can describe fully the properties of $a$-Si; we use a modified Stillinger-Weber potential, where the three-body contribution to the energy is enhanced by $50 \%$ with respect to the two-body term. This ad hoc modification was shown to produce good structural properties for amorphous silicon. ${ }^{2,10,4,18}$

After relaxation at zero pressure, the two 1000-atom configurations remain perfectly coordinated. The 4096-atom cell, less well relaxed, develops a few coordination defects: based on the first minimum in the RDF, it presents, respectively, $0.4 \%$ and $0.3 \%$ of threefold-and fivefold-coordinated atoms.

Table II presents the structural and energetic properties of the relaxed CRN's at zero pressure. For all configurations, the bond-angle distribution widens and the density decreases significantly compared to the Keating-relaxed structures. For the 1000-atom configurations, the local relaxation with the modified Stillinger-Weber potential did not result in a change of topology and the total energies are very low compared with previous models. ${ }^{19}$ We therefore expect that the structures will be stable with any reasonable potential.

Figure 4 shows a comparison of configuration $\mathrm{B}$ with experimental data obtained by Laaziri et al. on annealed $a$-Si samples prepared by ion bombardment. The agreement between the two is excellent except for some discrepancy in the height of the third-neighbor peak. Such an agreement must only be seen as a sign that the topology might be right, however: configurations differing widely in their topology can easily produce similar radial distribution functions. ${ }^{20}$ The same figure also presents the bond-angle distribution for configuration B relaxed with both the Keating and the modified Stillinger-Weber potentials. As expected for a perfectly coordinated configuration, the distribution is smooth and presents a single peak centered at the tetrahedral angle.

To compare with previous molecular dynamics studies, ${ }^{21}$ we have also relaxed our cells with the standard StillingerWeber potential, which is known to give an incorrect amorphous structure. After relaxation of configuration A, we find 17 atoms with fivefold coordination and three threefoldcoordinated ones; similar results are found with the two other networks. The resulting configurational energy, given in Table II, compares favorably with molecular dynamical results.

Contrary to the topological properties, which seem relatively independent of the details of the potential, we see that the ideal density of amorphous silicon compared with that of the crystal changes qualitatively as a function of the potential used. Configurations relaxed at zero pressure with the Keating potential show a reproducible densification by $2 \%$ while the modified Stillinger-Weber potential, also at zero pressure, leads to a structure which is up to $6 \%$ less dense. The latter results are in qualitative agreement with experiment ${ }^{22}$ and previous simulations using a similarly modified potential. ${ }^{10}$ Recently, Laaziri and collaborators have pointed to the lower density of $a$-Si as an explanation for the rela-
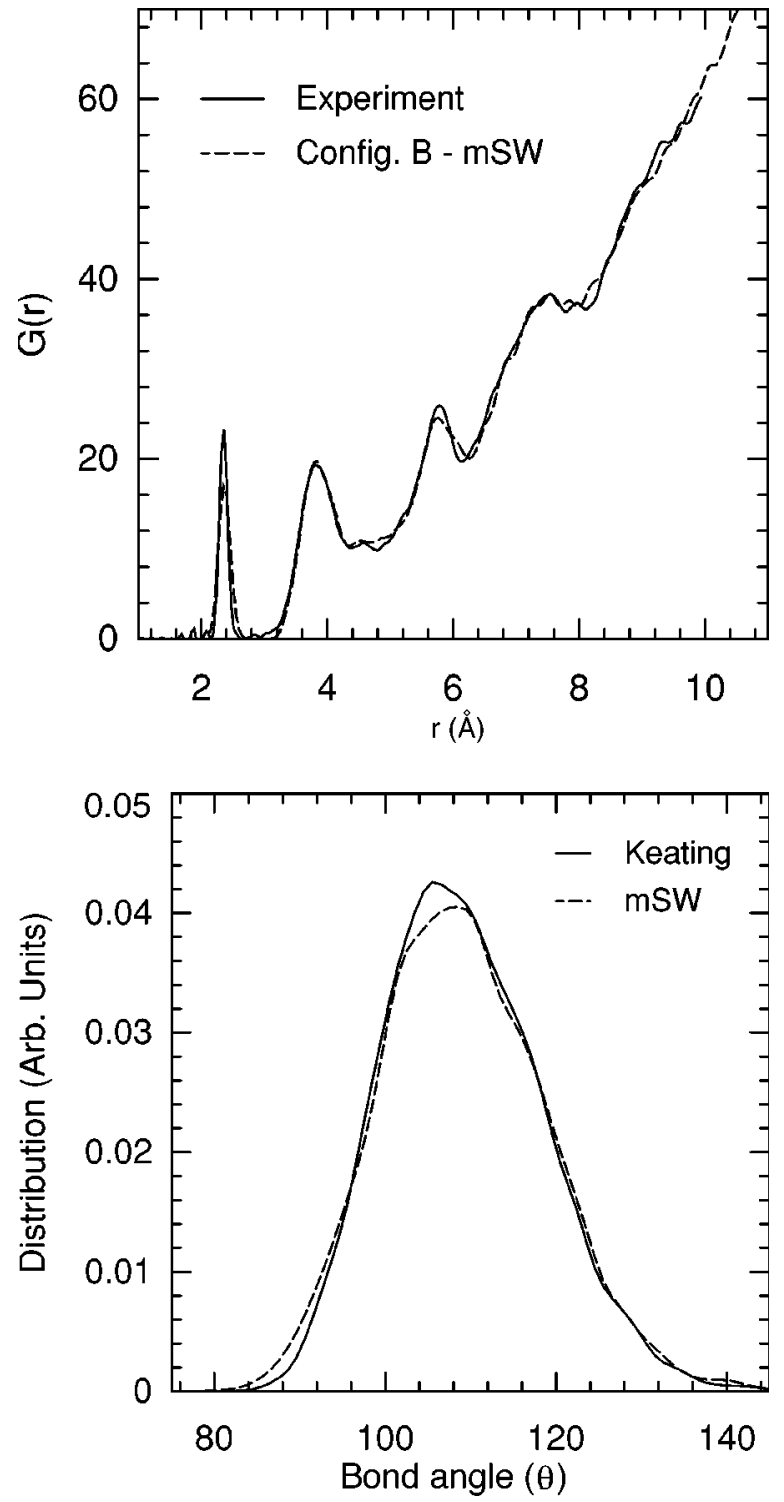

FIG. 4. Top: Radial distribution function for configuration B relaxed with the modified Stillinger-Weber potential. Solid line: experimental results from Ref. 12. To obtain a better fit, the computer-generated structure is scaled by a linear factor of 0.99 . Bottom: Bond-angle distribution for configuration B relaxed with Keating and the modified Stillinger-Weber potential. The curve is smoothed with a Gaussian of width $2^{\circ}$.

tively low coordination measured by $\mathrm{x}$-ray scattering. Our results, on the contrary, indicate that there is very little dependence between the density of the amorphous material and its topology, at least within the application of our two empirical potentials. A volume change at the percent level should therefore have very little impact on the topology and will reflect mostly some fine details of the real atomic interactions.

\section{CONCLUSIONS}

We have presented here modifications to the WootenWiner-Weaire algorithm that allows one to produce large 
high-quality continuous random networks without passing at all by a crystalline phase. The structural properties of the networks produced are excellent and they compare favorably with experiment. Moreover, the electronic density of states, obtained using ab initio interactions, ${ }^{16}$ show a wide and totally clean electronic band gap. The coordinates of the three configurations discussed here, as well as a 10000 -atom configuration under preparation, are available by request.

\section{ACKNOWLEDGMENTS}

We thank S. Roorda for providing us with his experimental data and S. Nakhmanson for computing the electronic density of states of configuration A. N.M. acknowledges stimulating discussion with D. A. Drabold and partial support by the National Science Foundation under Grant No. DMR-9805848.
*Email address: barkema@phys.uu.nl

†Email address: mousseau@ ohio.edu

${ }^{1}$ W.H. Zachariasen, J. Am. Chem. Soc. 54, 3841 (1932).

${ }^{2}$ K. Ding and H.C. Andersen, Phys. Rev. B 34, 6987 (1986); J.M. Holender and G.J. Morgan, J. Phys. Condens. Matter 3, 7241 (1991).

${ }^{3}$ S.J. Cook and P. Clancy, Phys. Rev. B 47, 7686 (1993).

${ }^{4}$ G.T. Barkema and N. Mousseau, Phys. Rev. Lett. 77, 4358 (1996).

${ }^{5}$ J.F. Justo, M. Z. Bazant, E. Kaxiras, V. V. Bulatov, and Y. S. Yip, Phys. Rev. B 58, 2539 (1998).

${ }^{6}$ K. Laaziri, S. Kycia, S. Roorda, M. Chicoine, J.L. Robertson, J. Wang, and S.C. Moss, Phys. Rev. Lett. 82, 3460 (1999).

${ }^{7}$ S. Roorda, W.C. Sinke, J.M. Poate, D.C. Jacobson, S. Dierker, B.S. Dennis, D.J. Eaglesham, F. Spaepen, and P. Fuoss, Phys. Rev. B 44, 3702 (1991).

${ }^{8}$ L. Guttman, W.Y. Ching, and J. Rath, Phys. Rev. Lett. 44, 1513 (1980).

${ }^{9}$ F. Wooten, K. Winer, and D. Weaire, Phys. Rev. Lett. 54, 1392 (1985); F. Wooten and D. Weaire, Solid State Phys. 40, 1 (1987).

${ }^{10}$ J.M. Holender and G.J. Morgan, J. Phys. Condens. Matter 3, 7241 (1991).
${ }^{11}$ B.R. Djordjević, M.F. Thorpe, and F. Wooten, Phys. Rev. B 52, 5685 (1995).

${ }^{12}$ K. Laaziri, S. Kycia, S. Roorda, M. Chicoine, J.L. Robertson, J. Wang, and S.C. Moss, Phys. Rev. B 60, 13520 (1999).

${ }^{13}$ See, for example, L.J. Lewis and N. Mousseau, Comput. Mater. Sci. 12, 210 (1998).

${ }^{14}$ P.N. Keating, Phys. Rev. 145, 637 (1966).

${ }^{15}$ D. Beeman, R. Tsu, and M.F. Thorpe, Phys. Rev. B 32, 874 (1985).

${ }^{16}$ O.F. Sankey and D.J. Niklewski, Phys. Rev. B 40, 3979 (1989); O.F. Sankey, D.A. Drabold, and G.B. Adams, Bull. Am. Phys. Soc. 36, 924 (1991).

${ }^{17}$ J.J. Dong and D.A. Drabold, Phys. Rev. Lett. 80, 1928 (1998).

${ }^{18}$ N. Mousseau and G.T. Barkema, Phys. Rev. B 61, 1898 (2000).

${ }^{19}$ N. Mousseau and G.T. Barkema, Phys. Rev. E 57, 2419 (1998).

${ }^{20}$ N. Mousseau and L.J. Lewis, Phys. Rev. Lett. 78, 1484 (1997); Phys. Rev. B 56, 9461 (1997).

${ }^{21}$ See, for example, R. Biswas, G.S. Grest, and C.M. Soukoulis, Phys. Rev. B 36, 7437 (1987); W.D. Luetdke and U. Landman, ibid. 37, 4656 (1988).

${ }^{22}$ J.S. Custer, M.O. Thompson, D.C. Jacobson, J.M. Poate, S. Roorda, and W.C. Sinke, Appl. Phys. Lett. 64, 437 (1994).

${ }^{23}$ L.J. Lewis and R.M. Nieminen, Phys. Rev. B 54, 1459 (1996). 